\title{
Immunohistochemical Expression of CD133 and LGR5 in Ulcerative Colitis-associated Colorectal Cancer and Dysplasia
}

\author{
SHINSUKE KAZAMA ${ }^{1,2}$, JUNKO KISHIKAWA ${ }^{2}$, TOSHIAKI TANAKA ${ }^{2}$, KEISUKE HATA ${ }^{2}$, \\ KAZUSHIGE KAWAI ${ }^{2}$, HIROAKI NOZAWA ${ }^{2}$ and SOICHIRO ISHIHARA ${ }^{2}$ \\ ${ }^{1}$ Division of Gastroenterological Surgery, Saitama Cancer Center, Saitama, Japan; \\ ${ }^{2}$ Division of Surgical Oncology, Department of Surgery, Faculty of Medicine, The University of Tokyo, Tokyo, Japan
}

\begin{abstract}
Background/Aim: Cluster of differentiation 133 (CD133) and leu cine-rich orphan G-protein-coupled receptor 5 (LGR5) are the most putative stem cell markers for colorectal cancer $(C R C)$, and are associated with poor prognosis of patients with CRC. However, the role of CD133 and LGR5 in the inflammation-dysplasia-carcinoma sequence has not been fully elucidated. We examined the expression of CD133 and LGR5 in ulcerative colitisassociated CRC (UC-CRC; $n=20)$ and UC-associated colorectal dysplasia $(n=16)$ by immunohistochemistry. Results: The rate of CD133-positive cases in UC-CRC was significantly higher than that in dysplasia $(p=0.026)$, but that of LGR5 expression was not. Moreover, LGR5 expression was significantly positively associated with p53 expression ( $p=0.03$ ), whereas $C D 133$ expression positively correlated with p53 expression, but not significantly $(p=0.10)$. Conclusion: CD133 may play an important role in tumor development in the context of the inflammationdysplasia-carcinoma sequence. LGR5-positive cancer stem cells may play a critical role in the development of UC-CRC, particularly upon loss of p53 function.
\end{abstract}

It is generally recognized that patients with extensive and long-standing ulcerative colitis (UC) have an increased risk of colorectal cancer (CRC). This relationship was first reported in 1925, wherein Crohn and Rosenberg documented a case of rectal carcinoma occurring in a patient with UC (1). More recently, Eaden et al. reported that the cumulative risk

This article is freely accessible online.

Correspondence to: Shinsuke Kazama, MD, Ph.D., Division of Gastroenterological Surgery, Saitama Cancer Center, 780, Komuro, Ina, Kitaadachi, Saitama, 362-0806, Japan. Tel: +81 487221111, Fax: +81487221129, e-mail: kaz-tky@umin.ac.jp

Key Words: CD133, LGR5, ulcerative colitis-associated colorectal cancer, p53, immunohistochemistry. of UC-associated CRC (UC-CRC) was $1.6 \%, 8.3 \%$, and $18.4 \%$ after 10,20 , and 30 years of disease duration, respectively (2). The cause of UC-CRC is considered to be the accumulation of inflammatory changes associated with colonic epithelial injury, repair, and regeneration, and the disease is believed to occur in an inflammation-dysplasiacarcinoma sequence. To ascertain tumor aggressiveness in the context of this sequence, p53 expression has been used $(3,4)$, However, there is a need for other biomarkers that can predict the degree of tumor malignancy.

Recent reports have shown that cancer stem cells (CSCs) are involved in tissue regeneration and carcinogenesis in sporadic CRC. From this hypothesis, it stands to reason that CSCs may contribute to the inflammation-dysplasiacarcinoma sequence. The CSC theory suggests that cancers are hierarchically organized, with only a small population of cancer cells maintained as CSCs, capable of both selfrenewal and differentiation. Over the past few years, several CSC surface markers have been identified in both hematological disorders (5) and solid tumors. Among these, cluster of differentiation 133 (CD133) and leucine-rich orphan G-protein-coupled receptor 5 (LGR5) are now thought to be the most putative surface markers for colorectal CSCs. The cell-surface marker CD133, also known as prominin-1, is a five-transmembrane glycoprotein with a molecular weight of $120 \mathrm{kDa}$, and is localized at membrane protrusions (6). Although its cellular function is not clear, two studies from 2007 reported CD133 to be a candidate marker for CSCs $(7,8)$. Specifically, Ricci-Vitiani et al. demonstrated that CD133-positive tumor-initiating cells were able to self-renew and differentiate, as well as reestablish tumor heterogeneity upon serial transplantation (7). In addition, O'Brien et al. showed that CD133-positive cells, but not CD133-negative ones, formed tumors when injected into immunodeficient mice (8). Moreover, results from two recent meta-analyses suggested that CD133 expression is significantly associated with shorter patient survival, and may play an important role in CRC progression $(9,10)$. Finally, in our recent study evaluating CD133 expression in 
colorectal polyps, we reported that the expression of this marker was associated with tumor differentiation (11).

LGR5 is a glycoprotein hormone receptor with a seventransmembrane domain, and is a reported target of the WNT- $\beta$-catenin signaling pathway, the latter of which is important for the maintenance of colonic crypts (12). LGR5 is considered to be a stem cell marker in the small intestine and colon, because LGR5 was demonstrated to be localized at the base of small intestinal crypts, and LGR5positive cells were found to be able to generate intestinal epithelial cells of multiple lineages (13). In addition, two previous meta-analyses demonstrated that LGR5 expression was associated with tumor progression and poor prognosis $(14,15)$.

Although CD133 and LGR5 have both been associated with advanced tumors, the roles of these markers in the inflammation-dysplasia-carcinoma sequence have not been fully elucidated. Therefore, the aim of this study was to examine the expression of CD133 and LGR5 in UC-CRC and UC-associated colorectal dysplasia using immunohistochemistry, and to identify the stage at which CD133 and LGR5 expression increases in this sequence.

\section{Materials and Methods}

Patient samples of UC-CRC and UC-associated colorectal dysplasia. A total of 20 patients with UC-CRC and 16 with UCassociated colorectal dysplasia were included in this study. In this study, there were no exclusion criteria. All patients were surgically treated at the University of Tokyo Hospital between February 1990 and January 2014. In this study, we examined the primary tumor (such as the most advanced lesion) in each case, even though multiple cancerous and dysplastic lesions were observed in a given colorectal specimen of many patients. Resected specimens were immediately fixed in $10 \%$ buffered formalin and embedded in paraffin. Pathological diagnoses of primary tumors and lymph nodes were confirmed with hematoxylin-and-eosin staining by the Department of Pathology at the University of Tokyo. UC-associated colorectal tumors were assessed in accordance with Inflammatory Bowel Disease/ Dysplasia Morphology Study Group criteria, and graded as lowgrade dysplasia (LGD), high-grade dysplasia (HGD), or CRC (16). The study protocol was approved by the University of Tokyo Graduate School of Medicine Ethics Committee on March 8, 2013 [Review Number 10056-(2)], and written informed consent was obtained from all patients.

CD133 and LGR5 immunohistochemical staining. In order to analyze CD133 and LGR5 expression, two 4- $\mu$ m-thick sections were immunohistochemically stained as described below. The sections were deparaffinized in xylene and hydrated through a graded series of ethanol, and endogenous peroxidases were blocked with $0.3 \%$ hydrogen peroxide in methanol for $25 \mathrm{~min}$. After washing with phosphate-buffered saline (PBS), heat-induced antigen retrieval was performed in $0.01 \mathrm{M}$ sodium citrate buffer ( $\mathrm{pH}$ 6.0) using an autoclave. After washing in PBS, non-specific proteins were blocked with $5 \%$ bovine serum albumin for $30 \mathrm{~min}$. Each slide
Table I. Clinicopathological features of patients with ulcerative colitis (UC)-associated colorectal cancer and dysplasia.

\begin{tabular}{|c|c|c|}
\hline Variable & & Value \\
\hline \multirow[t]{2}{*}{ Gender, $\mathrm{n}$} & Male & 22 \\
\hline & Female & 14 \\
\hline Age at UC onset, years & $\operatorname{Median} \pm$ SD & $33.0 \pm 13.8$ \\
\hline Age at operation, years & $\operatorname{Median} \pm$ SD & $50.0 \pm 13.5$ \\
\hline \multirow[t]{3}{*}{ Duration of UC } & $<10$ Years & 8 \\
\hline & $\geq 10$ to $<20$ Years & 12 \\
\hline & $\geq 20$ Years & 16 \\
\hline \multirow[t]{2}{*}{ Extent of inflammation, $\mathrm{n}$} & Total colitis & 35 \\
\hline & Left-sided colitis & 1 \\
\hline \multirow[t]{5}{*}{ Primary site of main tumor, $\mathrm{n}$} & Cecum & 1 \\
\hline & Transverse & 1 \\
\hline & Desending & 5 \\
\hline & Sigmoid & 7 \\
\hline & Rectum & 22 \\
\hline \multirow{6}{*}{ T-Stage of main tumor, $\mathrm{n}^{*}$} & Low-grade dysplasia & 4 \\
\hline & High-grade dysplasia & 12 \\
\hline & $\mathrm{T} 1$ & 7 \\
\hline & $\mathrm{T} 2$ & 1 \\
\hline & $\mathrm{T} 3$ & 8 \\
\hline & $\mathrm{T} 4$ & 4 \\
\hline \multirow[t]{6}{*}{ Histological type, $\mathrm{n}$} & Low-grade dysplasia & 4 \\
\hline & High-grade dysplasia & 12 \\
\hline & Well & 13 \\
\hline & Mod & 3 \\
\hline & Muc & 2 \\
\hline & Signet-ring cell & 2 \\
\hline \multirow{2}{*}{ Lymph node metastasis, $\mathrm{n}$} & Absent & 32 \\
\hline & Present & 4 \\
\hline
\end{tabular}

Well: Well-differentiated; Mod: moderately differentiated; Muc: mucinous; SD: standard deviation. *TMN Classification of Malignant Tumors, 7th edition.

was then incubated overnight at $4^{\circ} \mathrm{C}$ with primary anti-CD133 (AC133; Miltenyi Biotec, Auburn, CA, USA) and rabbit anti-LGR5 (Clone EPR3065Y; Epitomics, Burlingame, CA, USA) at a dilution of 1:100. After three washes with PBS, slides were then incubated with the Dako Envision kit (Dako, Carpinteria, CA, USA) according to the manufacturer's recommendations. After three washes with PBS, each slide was then incubated for 3 minutes in a chromogenic solution containing $2 \%$ 3,3'-diaminobenzidine tetrahydrochloride

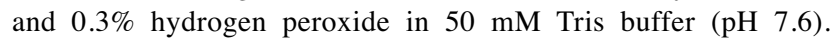
Mayer's/Lillie-Mayer's hematoxylin was used for counterstaining. Finally, the sections were dehydrated through a graded series of ethanolic solutions, incubated in xylene, and mounted. Renal tubules were used as positive controls, and for a negative control, antibodies were replaced with PBS.

Evaluation of CD133 and LGR5 immunostaining. CD133 positivity was defined as $>5 \%$ positively stained cancer cells, consistent with results from our previous study of CD133 expression in colorectal polyps and cancer $(11,17)$. Moreover, LGR5 positivity was defined as $>50 \%$ positively stained cancer cells, in accordance with our previous study of LGR5 expression in rectal cancer tissue after chemoradiotherapy (18). The sections were independently analyzed 
by two surgical pathologists (S.K. and J.K.) in a blinded fashion Any discrepancies were resolved by discussion. Subsequently, we analyzed the correlations between LGR5 or CD133 expression and patient clinicopathological features.

Statistical analysis. Correlations of CD133 and LGR5 expression with patient clinicopathological features were evaluated with the Chi-squared test, Fisher's exact test, or unpaired $t$-test, as appropriate. All statistical analyses were performed with JMP Pro 11.0.0 (SAS Institute, Cary, NC, USA). An association was considered significant when $p<0.05$.

\section{Results}

Clinical characteristics of patients with UC-CRC, and UCassociated colorectal dysplasia. The characteristics of the 36 patients are summarized in Table I. There were 22 male and 14 female patients, with a median age at UC onset of 33 years (range $=16-62$ years) and a median age at surgery of 50 years (range $=26-83$ years). Thirty-five patients $(97.2 \%)$ had total colitis, while one patient $(2.8 \%)$ had left-sided colitis. Among the 20 patients with UC-CRC, lymph node metastases were detected in four $(20.0 \%)$.

CD133 expression in UC-CRC and UC-associated colorectal dysplasia. CD133 expression was detected on the luminal surface but not in the cytoplasm of colorectal tumor glands. In some glands, intraglandular cellular debris was also CD133-positive (Figure 1). This expression pattern was consistent with previous reports of sporadic CRC $(11,17)$.

Evaluation of primary tumors revealed that 14 out of 36 cases $(39 \%)$ were CD133-positive; these included three HGD cases $(25 \%)$, and 11 UC-CRC cases $(55 \%)$. The rate of CD133 positivity in UC-CRC was significantly higher that in UC-associated colorectal dysplasia $(p=0.025)$ (Table II). No associations between CD133 expression and other clinicopathological factors, such as duration of UC, histological type, and the presence or absence of lymph node metastases, were observed. Finally, the rate of CD133positivity, as well as p53-positivity, in right colonitis tended to be higher than that in left, but not significantly ( $p=0.07$ and $p=0.10$, respectively).

LGR5 expression in UC-CRC and UC-associated colorectal dysplasia. Similarly to previous reports of sporadic CRC, LGR5 expression was detected in the cytoplasm of UC-CRC and UC-associated colorectal dysplasia cells (Figure 2). LGR 5 expression was detected in 28 cases $(78 \%)$, including two LGD cases (50\%), 10 HGD cases $(83 \%)$, and 16 UCCRC cases (80\%). No associations between LGR5 expression and tumor (pT) stage were found. Moreover, no associations between LGR5 expression and other patient clinicopathological features were detected; however, we did observe that LGR5 expression was significantly positively associated with p53 expression $(p=0.03)$ (Table II).

\section{Discussion}

In the present study, we examined the expression patterns of CD133 and LGR5 in UC-CRC and UC-associated colorectal dysplasia. The rate of CD133 positivity in UC-CRC was significantly higher than that in dysplasia $(p=0.026)$, but LGR5 expression was not associated with tumor grade. Although neither CD133 nor LGR5 expression was associated with other patient clinicopathological features, LGR5 expression was significantly positively associated with p53 expression ( $p=0.03$ ). Moreover, CD133 expression tended to be positively correlated with p53 expression, but not significantly $(p=0.10)$.

Many previous studies concerning associations between immunohistochemical expression of CSC markers, such as CD133 and LGR5, and CRC patient clinicopathological features and survival have been reported. In fact, a number of studies, including two meta-analyses, have shown reduced overall survival among patients with CD133-positive CRC (9, 10). It is well established that almost all CRCs arise from adenomas, in accordance with the adenoma-carcinoma sequence theory. Recently, we examined the expression of CD133 in adenomas to clarify the stage at which CD133 expression increases in this sequence (11). We demonstrated that $17.9 \%$ of colorectal adenomas were positive for CD133 expression by immunohistochemistry, and that CD133 expression was positively associated with the degree of adenoma differentiation. Therefore, these data suggest that CD133 may play an important role in tumor development. However, to the best of our knowledge, only one report has investigated the behavior and distribution of CD133 expression in UC-CRC and dysplasia, and the role of CD133 in the inflammation-dysplasia-carcinoma sequence has not been fully investigated. Specifically, Yasuda et al. investigated CD133 expression in UC-CRC and inflamed colonic epithelium using real-time reverse-transcription polymerase chain reaction, and demonstrated that the level of CD133 expression in inflamed colonic epithelium was significantly lower in patients with UC with a longer duration of disease than in those with a shorter duration (19). They also examined CD133 expression in a small number $(n=6)$ of UC-CRC cases. In the present study, we examined $20 \mathrm{UC}-\mathrm{CRC}$ cases and 16 cases of UC-associated colorectal dysplasia, and demonstrated that CD133 expression was associated with the degree of differentiation. These data are consistent with the proposed inflammation-dysplasia-carcinoma sequence, and suggest that CD133 may play an important role in tumor development in this context. Furthermore, our results indicate that evaluation of both CD133 and p53 expression by immunohistochemistry may be a useful diagnostic tool or biomarker for early detection of UC-CRC.

With respect to LGR5, previous studies, including two meta-analyses, demonstrated that LGR5 expression was 
in vivo $33: 1279-1284(2019)$

Table II. Cluster of differentiation 133 (CD133) and leucine-rich orphan G-protein-coupled receptor 5 (LGR5) expression in ulcerative colitis (UC)associated colorectal cancer and UC-associated colorectal dysplasia.

\begin{tabular}{|c|c|c|c|c|c|c|c|}
\hline \multirow[t]{2}{*}{ Variable } & & \multicolumn{3}{|c|}{ CD133 immunohistochemistry } & \multicolumn{3}{|c|}{ LGR5 immunohistochemistry } \\
\hline & & $\begin{array}{c}\text { Present } \\
(\mathrm{n}=14)\end{array}$ & $\begin{array}{l}\text { Absent } \\
(\mathrm{n}=22)\end{array}$ & $p$-Value & $\begin{array}{c}\text { Present } \\
(\mathrm{n}=28)\end{array}$ & $\begin{array}{c}\text { Absent } \\
(\mathrm{n}=8)\end{array}$ & $p$-Value \\
\hline \multirow[t]{2}{*}{ Gender, n (\%) } & Male & $8(36 \%)$ & $14(64 \%)$ & 0.69 & $19(86 \%)$ & 3 & 0.12 \\
\hline & Female & $6(43 \%)$ & $8(47 \%)$ & & $9(64 \%)$ & 5 & \\
\hline Age at UC onset, years & $\operatorname{Median} \pm$ SD & $35.5 \pm 23.6$ & $27.0 \pm 15.1$ & 0.89 & $33.0 \pm 26.7$ & $31.5 \pm 32.1$ & 0.76 \\
\hline Age at operation, years & Median \pm SD & $53.0 \pm 13.0$ & $49.0 \pm 14.0$ & 0.35 & $51.0 \pm 13.6$ & $49.0 \pm 13.8$ & 0.55 \\
\hline \multirow[t]{3}{*}{ Duration of UC } & $<10$ Years & $2(25 \%)$ & $6(75 \%)$ & 0.71 & $5(63 \%)$ & $337 \%)$ & 0.84 \\
\hline & $\geq 10$ to $<20$ Years & $5(42 \%)$ & $7(45 \%)$ & & $9(75 \%)$ & $3(25 \%)$ & \\
\hline & $\geq 20$ Years & $7(44 \%)$ & $9(56 \%)$ & & $14(88 \%)$ & $2(12 \%)$ & \\
\hline \multirow[t]{2}{*}{ Extent of inflammation, $\mathrm{n}(\%)$} & Total colitis & $13(37 \%)$ & $22(63 \%)$ & 0.20 & $27(77 \%)$ & $8(23 \%)$ & 0.59 \\
\hline & Left-sided colitis & $1(100 \%)$ & $0(0 \%)$ & & $1(100 \%)$ & $0(0 \%)$ & \\
\hline \multirow[t]{5}{*}{ Primary site of main tumor, $\mathrm{n}(\%)$} & Cecum & $1(100 \%)$ & $0(0 \%)$ & 0.07 & $1(100 \%)$ & $0(0 \%)$ & 0.73 \\
\hline & Transverse & $1(100 \%)$ & $0(0 \%)$ & & $1(100 \%)$ & $0(0 \%)$ & \\
\hline & Desending & $1(25 \%)$ & $4(75 \%)$ & & $4(80 \%)$ & $1(20 \%)$ & \\
\hline & Sigmoid & $4(57 \%)$ & $3(43 \%)$ & & $5(71 \%)$ & $2(29 \%)$ & \\
\hline & Rectum & $7(32 \%)$ & $15(68 \%)$ & & $17(77 \%)$ & $5(23 \%)$ & \\
\hline \multirow[t]{6}{*}{ pT Stage of main tumor, $\mathrm{n}(\%)$} & Low-grade dysplasia & $0(0 \%)$ & $4(100 \%)$ & $0.026^{*}$ & $2(50 \%)$ & $2(50 \%)$ & $0.72 *$ \\
\hline & High-grade dysplasia & $3(25 \%)$ & $9(75 \%)$ & & $10(83 \%)$ & $2(17 \%)$ & \\
\hline & $\mathrm{T} 1$ & $2(29 \%)$ & $5(71 \%)$ & & $4(57 \%)$ & $3(43 \%)$ & \\
\hline & $\mathrm{T} 2$ & $1(100 \%)$ & $0(0 \%)$ & & $1(100 \%)$ & $0(0 \%)$ & \\
\hline & $\mathrm{T} 3$ & $6(75 \%)$ & $2(25 \%)$ & & $7(88 \%)$ & $1(12 \%)$ & \\
\hline & $\mathrm{T} 4$ & $2(50 \%)$ & $2(50 \%)$ & & $4(100 \%)$ & $0(0 \%)$ & \\
\hline \multirow[t]{6}{*}{ Histological type, n (\%) } & Low-grade dysplasia & $0(0 \%)$ & $4(100 \%)$ & & $2(50 \%)$ & $2(50 \%)$ & \\
\hline & High-grade dysplasia & $3(25 \%)$ & $9(75 \%)$ & & $10(83 \%)$ & $2(17 \%)$ & \\
\hline & Well & $6(46 \%)$ & $7(54 \%)$ & 0.82 & $10(77 \%)$ & $3(23 \%)$ & 0.91 \\
\hline & Mod & $3(100 \%)$ & $0(0 \%)$ & & $3(100 \%)$ & $0(0 \%)$ & \\
\hline & Muc & $1(50 \%)$ & $1(50 \%)$ & & $2(100 \%)$ & $0(0 \%)$ & \\
\hline & Signet-ring cell & $1(50 \%)$ & $1(50 \%)$ & & $1(50 \%)$ & $1(50 \%)$ & \\
\hline \multirow{2}{*}{ Lymph node metastasis, n (\%) } & Absent & $11(34 \%)$ & $21(66 \%)$ & 0.15 & $24(75 \%)$ & $8(25 \%)$ & 0.26 \\
\hline & Present & $3(75 \%)$ & $1(25 \%)$ & & $4(100 \%)$ & $0(0 \%)$ & \\
\hline \multirow[t]{2}{*}{ p53 Immunohistochemistry, n (\%) } & Negative & $5(26 \%)$ & $14(74 \%)$ & 0.10 & $12(63 \%)$ & $7(37 \%)$ & 0.03 \\
\hline & Positive & $9(53 \%)$ & $8(47 \%)$ & & $16(94 \%)$ & $1(6 \%)$ & \\
\hline
\end{tabular}

Well: Well-differentiated; Mod: moderately differentiated; Muc: mucinous; SD: standard deviation. *Evaluation in UC-CRC versus UC-associated colorectal dysplasia.

associated with tumor progression and poor overall survival in sporadic CRC $(14,15)$. In addition, in colorectal adenomas, Takeda et al. reported that LGR5 expression in the lower crypt was positively associated with tumor grade (20). Moreover, Yang et al. showed that cytoplasmic expression of LGR5 increased with progression from normal mucosa to adenoma then to adenocarcinoma (21). However, no studies of immunohistochemical LGR5 expression have been reported in UC-CRC and dysplasia. Therefore, in the present study, we evaluated the association between LGR5 expression and patient clinicopathological factors. Associations between LGR5 expression and the degree of differentiation were not observed, inconsistent with the inflammation-dysplasiacarcinoma sequence. Specifically, the rates of LGR5 positivity were $50 \%(2 / 4)$ in LGD, $83 \%(10 / 12)$ in HGD, and $80 \%$ $(16 / 20)$ in UC-CRC. One possible explanation for this result is that increased expression of LGR5 may occur in early stages of UC-CRC tumorigenesis. As a result, further studies evaluating CD133 and LGR5 expression in a larger number of cases of UC-CRC and dysplasia are needed.

In the current study, we that showed LGR5 expression was positively associated with p53 expression $(p=0.03)$, and that CD133 expression tended to be positively correlated with $\mathrm{p} 53$ expression but not significantly $(p=0.10)$. Davidson et al. demonstrated that stem cell-specific p53 deletion greatly enhanced colonic tumor size and incidence in a mouse model of colitis-associated cancer (22). This study also suggested that the loss of p53 function in stem cells enables colonic tumor formation only when combined with DNA damage and chronic inflammation. Our results were consistent with these findings, suggesting that CSCs may play a critical role in the development of UC-CRC, particularly in the setting of p53 loss. However, further studies to this end are necessary. 
A

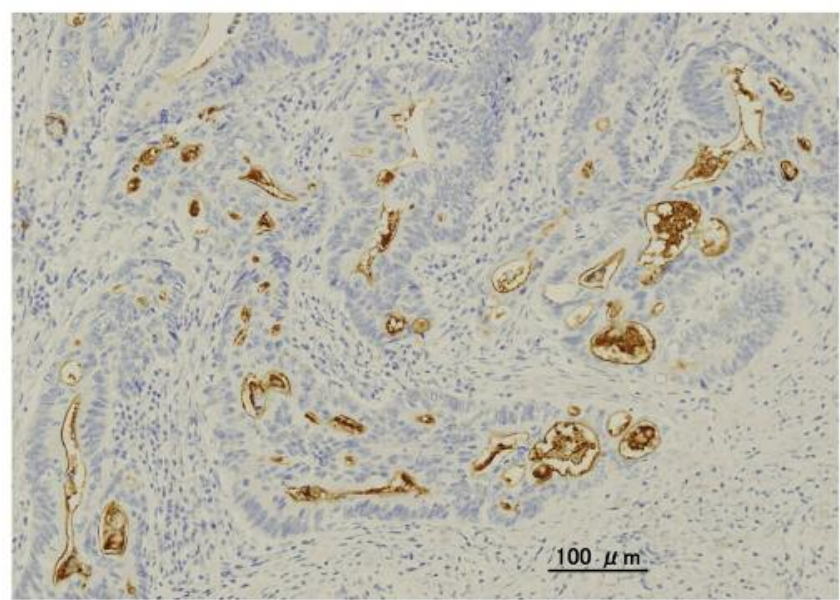

B

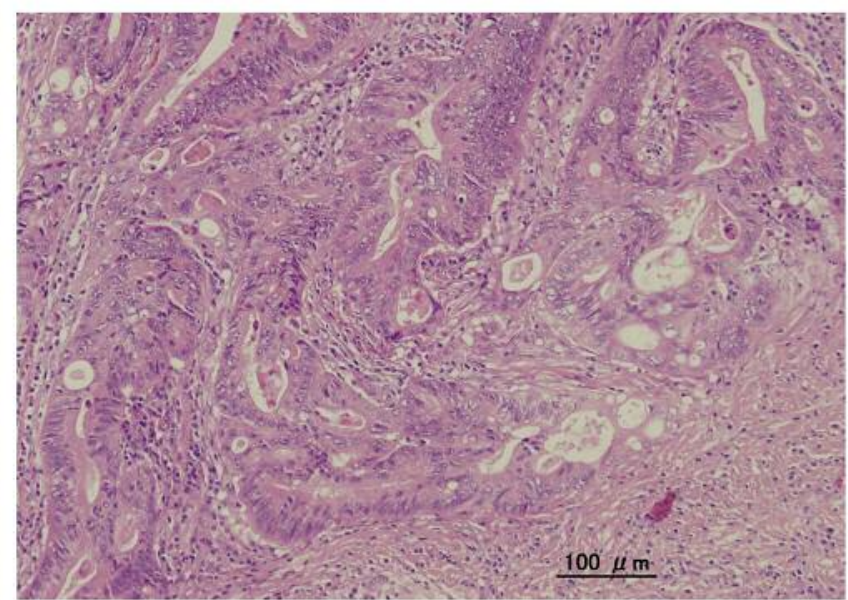

Figure 1. Immunohistochemical detection of cluster of differentiation 133 (CD133) in ulcerative colitis-associated colorectal cancer. A: Immunohistochemical CD133 expression was localized to the glandular-luminal surface of tumor epithelial cells and the intraglandular debris of shed tumor cells (original magnification: x40). B: Hematoxylin and eosin staining of a serial section (original magnification: x40).

A

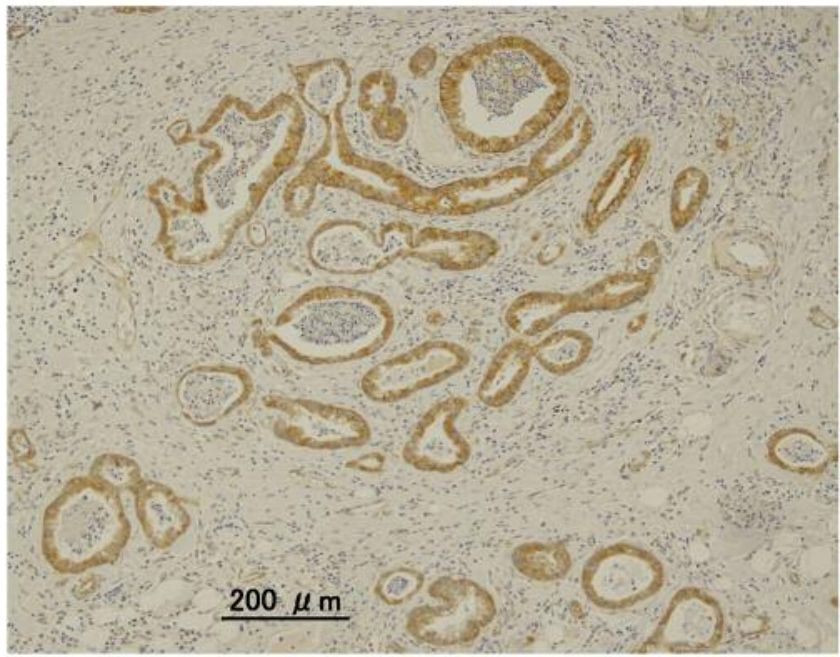

B

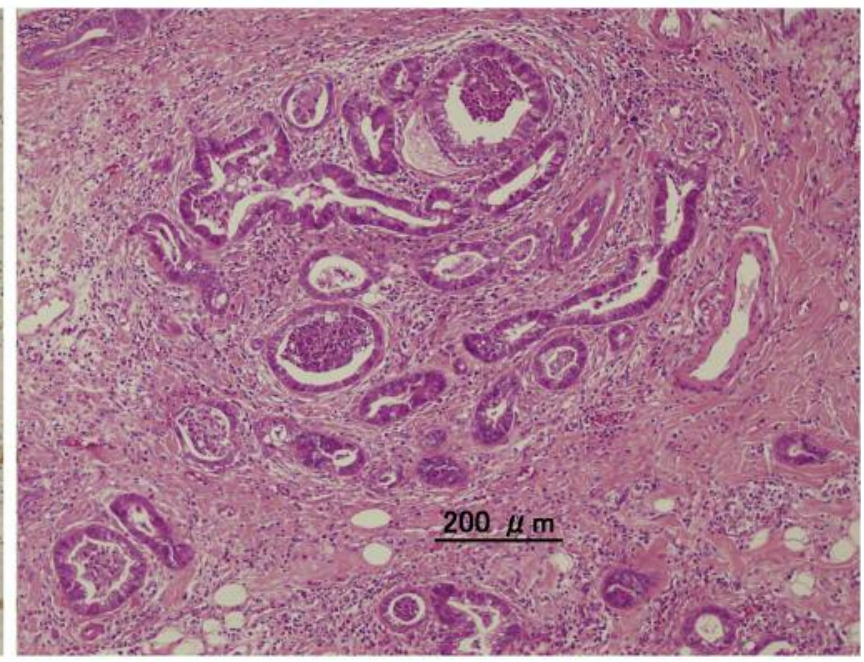

Figure 2. Immunohistochemical detection of leucine-rich orphan G-protein-coupled receptor 5 (LGR5) in ulcerative colitis-associated colorectal cancer. A: Immunohistochemical LGR5 expression was detected in the cytoplasm of tumor cells (original magnification: $\times 20$ ). B: Hematoxylin and eosin staining of a serial section (original magnification: $\times 20$ ).

Our data should be interpreted with caution due to the limitations of our study. Firstly, our study was limited by its retrospective nature, as well as the small number of patients included. Secondly, the analytic methods used to evaluate CD133 and LGR5 expression, including antibody used, method of evaluation, and cutoff scores for the definition of positive staining, have not been standardized. Further prospective, large-scale studies that use identical methodologies are needed.
In conclusion, we demonstrated that CD133 expression was positively associated with UC-associated colorectal dysplasia and UC-CRC tumor grade. Our findings suggest that CD133 may play an important role in tumor development in the context of the inflammation-dysplasia-carcinoma sequence. With respect to LGR5 expression, our data suggest that LGR5positive CSCs may play a critical role in the development of UC-CRC in the setting of p53 loss of function. 


\section{Conflicts of Interest}

The Authors declare that they have no conflicts of interest in regard to this study.

\section{Authors' Contributions}

SK contributed to the design and implementation of the study, and approval of the final version, JK was responsible for the recruitment of study participants, the clinical investigation and data collection. $\mathrm{TT}, \mathrm{KH}, \mathrm{KK}, \mathrm{HN}$, and SI were responsible for the study design, performed the experiments and thes tatistical analysis, and wrote the article. All Authors read and approved the final article.

\section{References}

1 Crohn BB and Rosenberg H: The sigmoidscopic picture of chronic ulcerative colitis (non-specific). Am J Med Sci 170: 220-228, 1925.

2 Eaden JA, Abrams KR and Mayberry JF: The risk of colorectal cancer in ulcerative colitis: a meta-analysis. Gut 48: 526-535, 2001. PMID 11247898.

3 Kobayashi K, Tomita H, Shimizu M, Tanaka T, Suzui N, Miyazaki $\mathrm{T}$ and Hara A: p53 Expression as a diagnostic biomarker in ulcerative colitis-associated cancer. Int J Mol Sci 18: 2017. PMID: 28621756. DOI: 10.3390/ijms18061284

4 Lu X, Yu Y and Tan S: p53 Expression in patients with ulcerative colitis associated with dysplasia and carcinoma: A systematic meta-analysis. BMC Gastroenterol 17: 111, 2017. PMID: 29070013. DOI: 10.1186/s12876-017-0665-y

5 Jones RJ and Armstrong SA: Cancer stem cells in hematopoietic malignancies. Biol Blood Marrow Transplant 14(Suppl 1): 12-16, 2008. PMID: 19122742. DOI: 10.1016/j.bbmt.2007. 10.012

6 Shmelkov SV, St Clair R, Lyden D and Rafii S: AC133/CD133/Prominin-1. Int J Biochem Cell Biol 37: 715719, 2005. PMID: 15694831. DOI: 10.1016/j.biocel.2004.08.010

7 Ricci-Vitiani L, Lombardi DG, Pilozzi E, Biffoni M, Todaro M, Peschle C and De Maria R: Identification and expansion of human colon-cancer-initiating cells. Nature 445: 111-115, 2007. PMID: 17122771. DOI: $10.1038 /$ nature05384

8 O'Brien CA, Pollett A, Gallinger S and Dick JE: A human colon cancer cell capable of initiating tumour growth in immunodeficient mice. Nature 445: 106-110, 2007. PMID: 17122772. DOI: $10.1038 /$ nature05372

9 Wang $\mathrm{K}, \mathrm{Xu}$ J, Zhang J and Huang J: Prognostic role of CD133 expression in colorectal cancer: A meta-analysis. BMC Cancer 12: 573, 2012. PMID: 23216926. DOI: 10.1186/1471-2407-12-573

10 Chen S, Song X, Chen Z, Li X, Li M, Liu H and Li J: CD133 expression and the prognosis of colorectal cancer: A systematic review and meta-analysis. PLoS One 8: e56380, 2013. PMID: 23409180. DOI: 10.1371/journal.pone.0056380

11. Kazama S, Kishikawa J, Kiyomatsu T, Kawai K, Nozawa H, Ishihara $\mathrm{S}$ and Watanabe T: Expression of the stem cell marker CD133 is related to tumor development in colorectal carcinogenesis. Asian J Surg 41: 274-278, 2018. PMID: 28190751. DOI: $10.1016 /$ j.asjsur.2016.12.002

12. Carmon KS, Gong X, Lin Q, Thomas A and Liu Q: R-spondins function as ligands of the orphan receptors LGR4 and LGR5 to regulate WNT/beta-catenin signaling. Proc Natl Acad Sci USA 108: 11452-11457, 2011. PMID: 21693646. DOI: 10.1073/ pnas. 1106083108
13 Barker N, van Es JH, Kuipers J, Kujala P, van den Born M, Cozijnsen M, Haegebarth A, Korving J, Begthel H, Peters PJ and Clevers $\mathrm{H}$ : Identification of stem cells in small intestine and colon by marker gene Lgr5. Nature 449: 1003-1007, 2007. PMID: 17934449. DOI: 10.1038/nature06196

14 Chen Q, Zhang X, Li WM, Ji YQ, Cao HZ and Zheng P: Prognostic value of LGR5 in colorectal cancer: A meta-analysis. PLoS One 9: e107013, 2014. PMID: 25192390. DOI: 10.1371/ journal.pone.0107013

15 Han Y, Xue X, Jiang M, Guo X, Li P, Liu F, Yuan B, Shen Y, Guo $\mathrm{X}$, Zhi Q and Zhao H: LGR5, a relevant marker of cancer stem cells, indicates a poor prognosis in colorectal cancer patients: A meta-analysis. Clin Res Hepatol Gastroenterol 39: 267-273, 2015. PMID: 25193236. DOI: 10.1016/j.clinre.2014.07.008

16 Riddell RH, Goldman H, Ransohoff DF, Appelman HD, Fenoglio CM, Haggitt RC, Ahren C, Correa P, Hamilton SR, Morson BC, Sommers SC and Yandley JH: Dysplasia in inflammatory bowel disease: standardized classification with provisional clinical applications. Hum Pathol 14: 931-968, 1983. PMID: 6629368.

17 Kishikawa J, Kazama S, Oba K, Hasegawa K, Anzai H, Harada Y, Abe H, Matsusaka K, Hongo K, Oba M, Yasuda K, Otani K, Nishikawa T, Tanaka T, Tanaka J, Kiyomatsu T, Hata K, Kawai K, Nozawa H, Yamaguchi H, Ishihara S, Sunami E, Ushiku T, Kitayama J, Fukayama M, Kokudo N and Watanabe T: CD133 Expression at the metastatic site predicts patients' outcome in colorectal cancer with synchronous liver metastasis. Ann Surg Oncol 23: 1916-1923, 2016. PMID: 26832881. DOI: 10.1245/ s10434-016-5099-1

18 Harada Y, Kazama S, Morikawa T, Murono K, Yasuda K, Otani K, Nishikawa T, Tanaka T, Kiyomatsu T, Kawai K, Hata K, Nozawa $\mathrm{H}$, Yamaguchi H, Ishihara, S and Watanabe T: Leucine-rich repeatcontaining $\mathrm{G}$ protein-coupled receptor 5 and $\mathrm{CD} 133$ expression is associated with tumor progression and resistance to preoperative chemoradiotherapy in low rectal cancer. Oncol Lett 14: 7791-7798, 2017. PMID:29250176. DOI: 10.3892/ol. 2017.7207

19 Yasuda H, Tanaka K, Okita Y, Araki T, Saigusa S, Toiyama Y, Yokoe T, Yoshiyama S, Kawamoto A, Inoue Y, Miki C and Kusunoki M: CD133, OCT4, and NANOG in ulcerative colitisassociated colorectal cancer. Oncol Lett 2(6): 1065-1071, 2011.

20 Takeda K, Kinoshita I, Shimizu Y, Matsuno Y, Shichinohe T and Dosaka-Akita H: Expression of LGR5, an intestinal stem cell marker, during each stage of colorectal tumorigenesis. Anticancer Res 31: 263-270, 2011. PMID: 22848268. DOI: 10.3892/ol.2011.415

21 Yang B, Mao L, Li Y, Li Q, Li X, Zhang Y and Zhai Z: betacatenin, leucine-rich repeat-containing $G$ protein-coupled receptor 5 and GATA-binding factor 6 are associated with the normal mucosa--adenoma-adenocarcinoma sequence of colorectal tumorigenesis. Oncol Lett 15: 2287-2295, 2018. PMID: 29434936. DOI: 10.3892/ol.2017.7566

22 Davidson LA, Callaway ES, Kim E, Weeks BR, Fan YY, Allred $\mathrm{CD}$ and Chapkin RS: Targeted deletion of p53 in Lgr5-expressing intestinal stem cells promotes colon tumorigenesis in a preclinical model of colitis-associated cancer. Cancer Res 75: 5392-5397, 2015. PMID: 26631266. DOI: 10.1158/0008-5472.CAN-15-1706

Received April 29, 2019

Revised June 11, 2019

Accepted June 13, 2019 